\title{
Video-level Multi-model Fusion for Action Recognition
}

\author{
Xiaomin Wang \\ College of Computer Science and \\ Technology, China Univ. of \\ Petroleum (EastChina) \\ Qingdao, Shandong, China \\ m17071007@s.upc.edu.cn \\ Philip S.Yu \\ Department of Computer Science, \\ Univ. of Illinois at Chicago \\ United States \\ psyu@uic.edu
}

\author{
Junsan Zhang* \\ College of Computer Science and \\ Technology, China Univ. of \\ Petroleum (EastChina) \\ Qingdao, Shandong, China \\ zhangjunsan@upc.edu.cn \\ Jie Zhu \\ Department of Information \\ Management, The National Police \\ Univ. for Criminal Justice \\ China \\ arthurzhujie@163.com
}

\author{
Leiquan Wang \\ College of Computer Science and \\ Technology, China Univ. of \\ Petroleum (EastChina) \\ Qingdao, Shandong, China \\ richiewlq@gmail.com \\ Haisheng Li \\ Beijing Key Laboratory of Big \\ Data Technology for Food \\ Safety,Beijing Technology and \\ Business Univ. \\ China \\ lihsh@th.btbu.edu.cn
}

\begin{abstract}
The approaches based on spatio-temporal features for video action recognition have emerged such as two-stream based methods and 3D convolution based methods. However, current methods suffer from the problems caused by partial observation, or restricted to single information modeling, and so on. Segment-level recognition results obtained from dense sampling can not represent the entire video and, therefore lead to partial observation. And a single model is hard to capture the complementary information on spacial, temporal and spatio-temporal information from video at the same time. Therefore, the challenge is to build the video-level representation and capture multiple information. In this paper, a video-level multi-model fusion action recognition method is proposed to solve these problems. Firstly, an efficient videolevel $3 \mathrm{D}$ convolution model is proposed to get the global information in the video which assembling segment-level 3D convolution models. Secondly, a multi-model fusion architecture is proposed for video action recognition to capture multiple information. The spatial, temporal and spatio-temporal information are aggregate with SVM classifier. Experimental results show that this method achieves the state-of-the-art performance on the datasets of UCF-101(97.6\%) without pre-training on Kinetics.
\end{abstract}

\footnotetext{
${ }^{*}$ Corresponding author
}

Permission to make digital or hard copies of all or part of this work for personal or classroom use is granted without fee provided that copies are not made or distributed for profit or commercial advantage and that copies bear this notice and the full citation on the first page. Copyrights for components of this work owned by others than ACM must be honored. Abstracting with credit is permitted. To copy otherwise, or republish, to post on servers or to redistribute to lists, requires prior specific permission and/or a fee. Request permissions from permissions@acm.org.

CIKM '19, November 3-7, 2019, Beijing, China

(C) 2019 Association for Computing Machinery.

ACM ISBN 978-1-4503-6976-3/19/11 . .\$15.00

https://doi.org/10.1145/3357384.3357935

\section{CCS CONCEPTS}

- Computing methodologies $\rightarrow$ Activity recognition and understanding; Neural networks; Classification and regression trees.

\section{KEYWORDS}

action recognition; video-leval recognition; 3D convolution; multi-model fusion

\section{ACM Reference Format:}

Xiaomin Wang, Junsan Zhang, Leiquan Wang, Philip S.Yu, Jie Zhu, and Haisheng Li. 2019. Video-level Multi-model Fusion for Action Recognition. In The 28th ACM International Conference on Information and Knowledge Management (CIKM '19), November 3-7, 2019, Beijing, China. ACM, New York, NY, USA, 10 pages. https://doi.org/10.1145/3357384.3357935

\section{INTRODUCTION}

Automatically recognizing the action of a video is an important and fundamental topic in artificial intelligence and compute vision. However, video action recognition is a challenging task, which involves a comprehensive understanding of spatial, temporal and spatio-temporal information. Considerable progresses have been made in action recognition such as two-stream based model and 3D convolution model.

Two-stream ConvNet architecture [34] which incorporates spatial and temporal networks was first posed by Simonyan, et al. The spatial stream performs action recognition from still video frames, while the temporal stream is trained to identify human motion from motion information of the dense optical flow. It appears that training a temporal ConvNet on optical flow is significantly better than training on raw stacked frames. However, the two-stream model trains two networks independently, which ignored the relationship between temporal and spatial. Static appearance information and motion information do not exist independently, and there is significant correlation between static appearance and motion information. 
3D Convolutional Neural Networks [18] extracts features from both the spatial and the temporal dimensions by performing $3 \mathrm{D}$ convolutions and captures the motion information encoded in multiple adjacent frames. 3D ConvNets create hierarchical representations of spatio-temporal information with spatio-temporal filters. They try to obtain spatio-temporal information using a set of consecutive frames. Dense sampling on video frames is widely used to achieve high accuracy for action recognition. One issue with dense sampling is that the slight variations among frames can lead to information redundancy. The following problem is that more parameters are needed during the training process. Traditional 3D convolution methods $[5,7,11,18,31-33]$ randomly select a continuous frame from a video as input( 8 frames, 16 frames, 64 frames or more frames). It is obvious that extracting more frames can result in better outcome. But this makes them hard to train due to the extensive parameters. Moreover, compared to traditional 3D convolutions, long-term 3D convolutions [33] reduce the spatial resolution of input frames to relieve memory consumption, from which finer cues are easily lost. A direct solution to this problem is to divide the entire video into some segments. However, the segments can not represent the entire video. In most cases it leads to a inaccurate result. As shown in Figure.1(a), the front part of the diving is similar to jumping and the last part of the action is very similar to swimming which is likely to cause confusion. In this situation, how to aggregate the segmentlevel representation to represent the whole video needs to be seriously treated.

$3 \mathrm{D}$ convolution is supposed to extract spatio-temporal information. However, the static RGB and optical flow still provides more detailed spatial and temporal information that is also beneficial for action recognition. Temporal and spatial recognition streams are complementary so that two-stream model[10, 19, 20, 34] was shown to get good performance on existing benchmarks. Figure.1(b) provides examples of predictions from different models, showing how the importance of multi-model fusion. The prediction of $3 \mathrm{D}$ convolution is "blowing candles" which is not accurate. But the spatial stream which is input by only static RGB image, captures some important appearance features such as candles, etc, thus gets the right results. From a multi-model perspective, employing multi-model features can also be a better solution for video action recognition due to the complementariness among spatial, temporal and spatio-temporal information. Based on the above observations, it is necessary to choose an optimal fusion strategy to overcome the shortcomings of 3D CNNs and two-stream model.

In general, the major disadvantages of the present and commonly used action recognition methods are: (1)The integrity of action sequence. Consecutive frames are used as input in $3 \mathrm{D}$ convolutional model to capture the information from more frames, however, it still cannot represent the global information in a video. A complete action sequence can fill the entire video. (2)The comprehensiveness of multi-dimensional information. Some information from the relationship between temporal and spatial is ignored by Two-stream ConvNet architecture while some detail of spatial and temporal information is neglected by $3 \mathrm{D}$ convolution model.

To address these problems, a new video-level multi-model fusion method for action recognition is proposed in this paper. Spatial, temporal and spatio-temporal information are fully exploited to get a comprehensive understanding on action recognition. The lightweight video-level 3D convolution network is built which combines segment-level 3D CNNs into video-level supervision to overcome the confusion caused by partial observation. Compared to the traditional 3D CNNs, the proposed lightweight video-level 3D convolution network reduce the number of parameters, and therefore reduce the training complexity. A global 3D convolution for video prediction is aggregated by averaging several segment-level predict scores. In addition, a linear SVM(support vector machine) classifier is trained to fuse the softmax scores from videolevel two-stream model and video-level 3D convolution model due to the complementariness among spatial, temporal and spatio-temporal information.

To summarize, the main contributions of this paper are as follows:

(a)An efficient video-level 3D convolution model by assembling segment-level 3D convolution model is proposed to get the global information in the video.

(b)A multi-model fusion architecture is proposed for video action recognition by aggregating spatial, temporal and spatiotemporal information with Support Vector Machine.

(c)Comprehensive experiments are conducted on the standard UCF-101 datasets. The results show that the proposed method has achieved competitive performance compared with the related video action recognition methods.

\section{RELATED WORK}

\subsection{Two-stream model}

Simonyan et al. [34] design the two-stream CNNs based on RGB data and optical flow. They show that the accuracy of action recognition is significantly boosted even by simply aggregating probability scores, which indicates that optical flow provides high-quality motion information. This framework extracts short snippets over a long video sequence with a sparse sampling scheme, where the samples distribute uniformly along the temporal dimension. Thereon, a segmental structure is employed to aggregate information from the sampled snippets. In this sense, temporal segment networks are capable of modeling long-range temporal structure over the whole video.Karpathy et al. [20] explore several approaches for fusing information over time based on appearance CNN. Wang et al. [34] extract two-stream CNNs along dense trajectories. Many methods based on two-stream CNNs have been proposed to improve the accuracy of action recognition. Feichtenhofer et al.[10] studied a number of ways of fusing CNNs both spatially and temporally in order to best take advantage of this spatio-temporal information . They also combined two-stream CNNs with ResNets[19] to show that the ResNet architecture is effective for action recognition with $2 \mathrm{D}$ CNNs[10].Naveed et al. [25]creat a fusion of features 


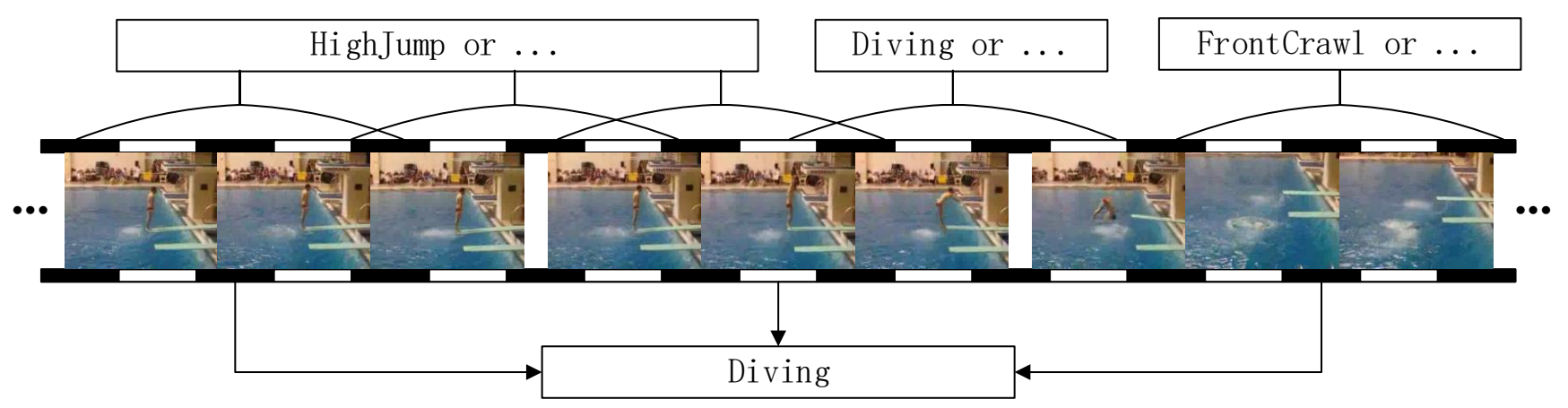

(a)

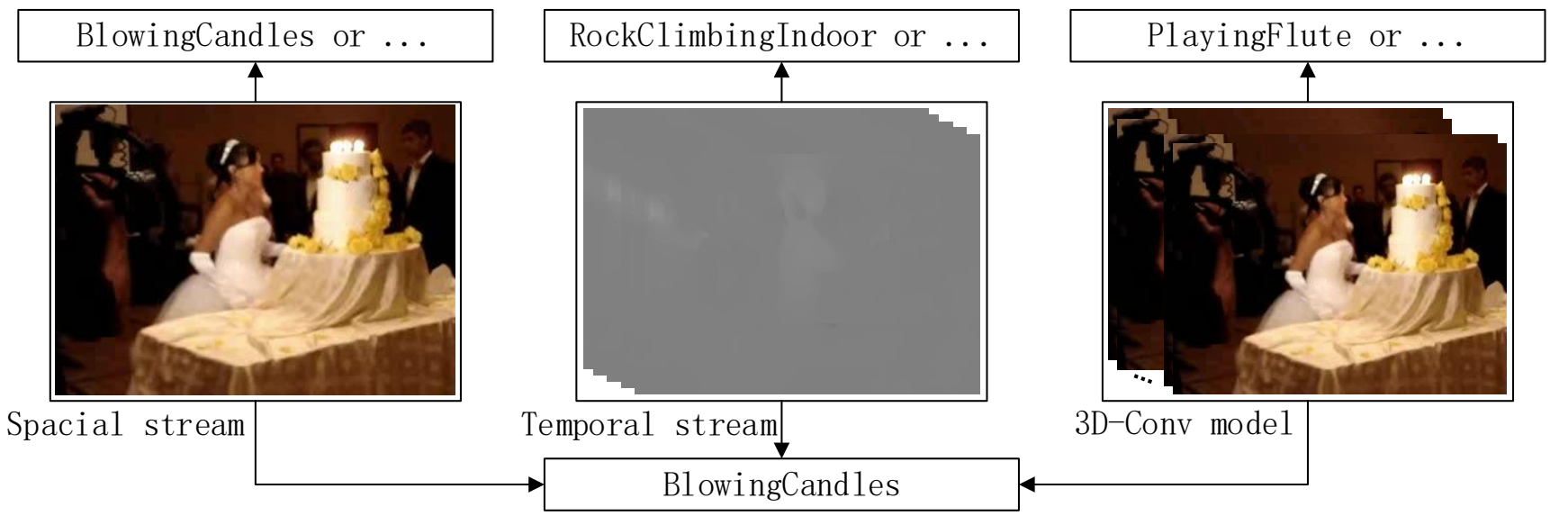

(b)

Figure 1: (a)The predictions of segment-level and video-level of the video of classes "diving" from UCF-101. Global observation is better than partial observation. (b) The predictions from different models. There are Complementarities between multiple models.

by incorporating flow-based, spatio-temporal and interest points based features which give better results.

Among these approaches, RGB images and optical flow stacks are used as the inputs of two separate models respectively, which demonstrates the deficiency of spatio-temporal information from 2D convolution. However, computing flow from video frames is very time consuming. Recent works directly leverage the motion vectors and residuals readily available in the compressed video to represent motion at no cost.

A number of approaches that utilize the information present in the compressed video domain have been proposed. Zhang et al $[2,40]$. replace the optical flow stream in two-stream methods by a motion vector stream. CoViAR [36] is proposed to exploit all data modalities in compressed videos, i.e. RGB I-frames, motion vectors and residuals to bypass RGB frame decoding. Motion representation and optical flow estimation are also used to replace the optical flow.

Optical flow estimation methods explicitly model the displacement at each pixel between successive frames [3, 15, 29,
39]. CNNs have successfully been trained to estimate the optical flow, including FlowNet [6, 16], SpyNet[26] and PWCNet[30]. And MPI Sintel [4] and KITTI 2015 [21] achieve low End-Point Error (EPE) on challenging benchmarks. Im2Flow work [12] also shows optical flow can be hallucinated from still images. [27] shows that accuracy of optical flow does not strongly correlate with accuracy of video recognition. Fan et al. [9] proposed to transform TV-L1 optical flow algorithm into a trainable sub-network, which can be jointly trained with downstream recognition network.

\subsection{D Convolutional model}

Most aforementioned deep learning-based methods treat video as a frame or optical flow image sequence for video representation learning while leaving the temporal evolution across consecutive frames not fully exploited. To tackle this problem, 3D Convolutional Neural Networks [18] was first posed by $\mathrm{Ji}$, et al to extract features from both the spatial and the temporal dimensions. Tian et al. [32] extend the 2D deformable part model [11] to 3D space-temporal volumes 
for action localization to learn discriminative features along both spatial and temporal dimensions. Another method to obtain motion information is C3D(Tran et al. 2015)[7], which is a natural extension of $2 \mathrm{D}$ CNNs. C3D relies on 3D convolution kernels to capture spatio-temporal information. The out-of-the-box C3D feature has been widely employed in many subsequent works on action recognition and detection. Varol et al. [33] found that expanding the temporal length of inputs for 3D CNNs can achieve better results and that using optical flows as inputs can outperform RGB inputs. Carreira et al. (I3D)[5] incorporated the Inception architecture [31] into 3D CNNs, filters and pooling kernels of very deep image classification ConvNets are expanded into 3D, making it possible to learn seamless spatio-temporal feature extractors from video while leveraging successful ImageNet architecture designs and even their parameters. Rabiee, et al.[25] propose a novel tracklet-based model for detection and localization of crowd behavior, which is based on the orientation and magnitude of short trajectories extracted by tracking interest points in spatio-temporal 3D patches.

One issue with these 3D Conv models is that they have many more parameters than 2D ConvNets because of the additional kernel dimension, and this makes them harder to train. Zhou et al. [38] propose a Mixed Convolutional Tube (MiCT) that integrates $2 \mathrm{D} \mathrm{CNN}$ with the $3 \mathrm{D}$ convolution module to generate deeper and more informative feature maps, while reducing training complexity in each round of spatio-temporal fusion.

As mentioned before, all these 3D CNNs for action recognition applies 3D convolutions on a short video clip with consecutive frames (typically 16). However, all these approaches build the clip-level features instead of the video-level representations, thus suffer from the confusion caused by partial observation training.

\subsection{Long-term video-level prediction}

Based on deep learning, several video-level representation learning methods have emerged recently. CNN methods excel at capturing short-term patterns in short, fixed-length videos, but it remains difficult to directly capture long-term interactions in long variable-length videos. Recently, several works focused on modeling long-term temporal structure with ConvNets. Recurrent neural networks, particularly long short-term memory (LSTM) [13]ones, have been considered to model long-term temporal interactions in video classification. $\mathrm{Ng}$ et al. [22] proposed two stream LSTMs for higher-accuracy video classification over longer time periods.Xiang Long et al. [37] propose a simple and efficient attention mechanism that effectively aids the training of the RNN model whitch architecture build upon a bidirectional LSTM. Limin Wang et al. [35] proposed Temporal Segment Networ(TSN) witch the class scores of different snippets are fused by an the segmental consensus function to yield segmental consensus to get a video-level prediction. [23] proposed Deep networks with Temporal Pyramid Pooling (DTPP), an end-to-end videolevel representation learning approach. A temporal pyramid pooling (TPP) layer is placed on top of them to aggregate several frame-level features into a video-level representation.

Moreover, in order to relieve memory consumption, longterm 3D convolutions[33] have reduce the spatial resolution of input frames, which result in the losing of finer cues.

\section{PROPOSED METHOD}

The architecture of proposed method(video-level multi-model fusion for action recognition) is shown in Figure. 2. Each video is firstly divided into $\mathrm{K}$ segments of equal durations. Then a set of data which including a single frame, stacked optical flow and continuous frames are sampled from each segment. The input of the architecture expressed as $V=\left\{V^{\alpha}, V^{\beta}, V^{\gamma}\right\}$ and the output of the architecture expressed as $o=O(V)$. Here $V^{\alpha}, V^{\beta}, V^{\gamma}$ denote the single frame, stacked flow and continuous frames respectively. $V^{\alpha}, V^{\beta}$ and $V^{\gamma}$ are enter into three separate model. And get the video-level multi-model fusion action recognition result $o$ finally. In this section, videolevel 3D convolution model are firstly introduced(Sec. 3.1). Then multi-model fusion are reported(Sec . 3.2). Finally, the training details of the structure are introduced(Sec. 3.3).

\subsection{Video-level 3D convolution model}

A lightweight video-level model is built based on 3D convolution model to overcome the confusion caused by partial observation. The video is divided into multiple segments as in TSN[35]. Then a video-level predictions are got by simply averaging the scores of each segment.

Firstly, in order to reduce redundant information, this framework extracts short snippets over a long video sequence with a sparse sampling scheme, rather than takes a bunch of consecutive frames as input rudely. So that the proposed 3D convolution based model can grasp the global informations, and do not increase the number of parameters at the same time. For each video, 5 frames are extracted per second instead of 25 frames. So there will be some obvious motion changes between adjacent frames.

Each video is divided into $\mathrm{K}$ segments. 16 consecutive frames are randomly sample from each segment, and respectively input into the $3 \mathrm{D}$ convolution model. The multiple segment-level scores from $\mathrm{K}$ segments are then averaged to get one video-level score. In the TSN[35], it has been proved that the maximum efficiency can be achieved when $\mathrm{K}$ is set to 3 . Increasing the value of $\mathrm{K}$ will increase the accuracy in a limited way, but increase the computing. So $\mathrm{K}$ is set to 3 when making predictions in our experiments. Of course, due to the large difference in video length, the number of frames which are extracted from some videos is less than $48(\mathrm{~K} \times 16)$. For a satisfactory overall effect, the last frame is repeatedly applied to fill up the gap.

It is worth noting that the $K$ is set to 1 in the process of training to reduce training costs and set to 3 in the process of testing to get video-level predictions in our experiments. In other words, 16 consecutive frames are used as input for training for each video. Sampling on the entire video randomly can also obtain consecutive frames at different locations during 


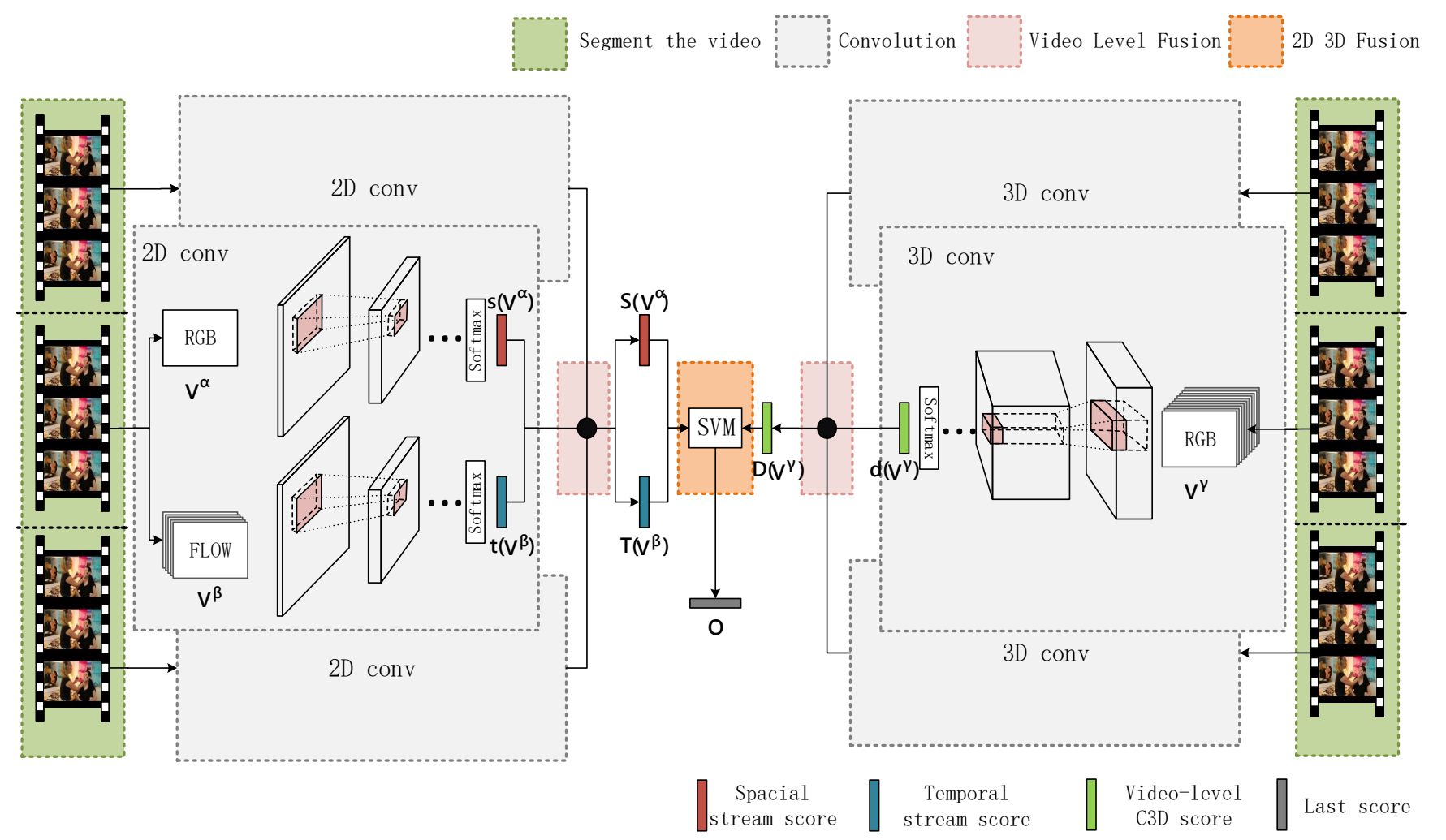

Figure 2: Multi-model fusion based video-level action recognition for video: $2 \mathrm{D}$ convolution model on the left and $3 \mathrm{D}$ convolution model on the right. One input video is divided into $\mathrm{K}$ (we take $K=3$ In this article) segments and a short snippet is randomly selected from each segment. The class scores of different snippets are fused to acquire a video-level prediction. Finally, a SVM classifier is trained to fuse the softmax scores from video-level two-stream model.

training and get good performance in segment-level prediction. However, video-level predictions are required during testing precess by averaging the scores of each segment-level action recognition.

A structure which is similar to $\mathrm{C} 3 \mathrm{D}[7]$ is adopted to capture the spatio-temporal information, which has 8 convolutional layers, 5 pooling layers and 2 fully connected layers. The size of the is $16 \times 112 \times 112$. And the model was pre-trained on the sports1M dataset. Compared to the traditional C3D, the proposed method has better effect without increasing the complexity and the number of parameters during the training.

As we can see in Figure. 2, this network takes the input $V=\left\{V^{\alpha}, V^{\beta}, V^{\gamma}\right\}$ and output the specific action class $o=$ $O(V)$ which can be formulated as

$$
\begin{aligned}
& S\left(V^{\alpha}\right)=\frac{1}{K}\left(\sum_{k=1}^{K} s^{k}\left(V^{\alpha}\right)\right) \\
& T\left(V^{\beta}\right)=\frac{1}{K}\left(\sum_{k=1}^{K} t^{k}\left(V^{\beta}\right)\right)
\end{aligned}
$$

$$
D\left(V^{\gamma}\right)=\frac{1}{K}\left(\sum_{k=1}^{K} d^{k}\left(V^{\gamma}\right)\right)
$$

The numbers of segments divided by video represented by $\mathrm{K}$ and $\mathrm{k}$ represents the $k^{t h}$ segment. $s(\cdot), t(\cdot)$ and $d(\cdot)$ represent the spatial stream model, temporal stream model and 3D convolution model. $s^{k}\left(V^{\alpha}\right)$ is the scores of spatial stream model which takes a single frame sampled from the $k^{t h}$ segment as input. $t^{k}\left(V^{\beta}\right)$ is the scores of temporal stream model which takes stacked flow sampled from the $k^{\text {th }}$ segment as input. And $d^{k}\left(V^{\gamma}\right)$ is the scores of 3D convolution model, which takes continuous frames sampled from the $k^{\text {th }}$ segment as input. The $S(\cdot), T(\cdot)$ and $D(\cdot)$ represent the video-level scores of these three models.

\subsection{Multi-model fusion}

In order to aggregating spatial, temporal and spatio-temporal informations, a method is proposed by combining 2D convolution with 3D convolution. A SVM classifier is trained to fuse the softmax scores from video-level two-stream model and video-level 3D convolution model. The spatial and temporal streams which take RGB images and optical flow stacks as input respectively can obtain the spacial and temporal 
information of the video. And the video-level 3D convolution model, take multiple frames as input, and obtain the spatio-temporal information of the video.

A SVM classifier is trained which takes the $S(\cdot), T(\cdot)$ and $D(\cdot)$ as input to fuse the three models. The $S(\cdot), T(\cdot)$ and $D(\cdot)$ represent the spatial stream model, temporal stream model and 3D convolution model.

$$
O(V)=S V M\left(S\left(V^{\alpha}\right) \oplus T\left(V^{\beta}\right) \oplus D\left(V^{\gamma}\right)\right)
$$

$\oplus$ represent adding together a series of softmax scores from different models. A multi-class linear SVM on stacked softmax scores is training then. The classification accuracy of different kernel function of SVM based on linear, polynomial, $\mathrm{RBF}$ ( Gaussian radial basis function ), etc were compared, the results of experiment show that the performance of polynomial kernel function is much better. The Polynomial kernel is a non-stationary kernel. The polynomial kernel function can map the low-dimensional input space to the feature space of high latitude, and polynomial kernels are well suited for problems where all the training data is normalized. And polynomial kernel is set to 7 . The polynomial kernel of degree 1 leads to a linear separation, and higher-degree polynomial kernels allow a more flexible decision boundary.

The $2 \mathrm{D}$ convolution model get video-level predictions by average the scores of each segment as lightweight video-level $3 \mathrm{D}$ convolution model. For $2 \mathrm{D}$ convolution(spatial stream model and temporal stream model which is mentioned above), a structure which is similar to TSN[35] is adopted. And the BN-Inception(Inception with Batch Normalization) [17] is chosen as building block to get a balance between accuracy and efficiency. The pre-trained TSN[35] models provided by Xiong are used. For spatial stream, each input frame size is $224^{*} 224^{*} 3$. For temporal stream, each input frame size is $224^{*} 224^{*} 10$, which is the stack of 5 consecutive optical flow fields.

The softmax scores are fused using either weighted summation method or SVM classifier for fusion. Weighted method of data fusion is a simple and effective method. And the weighted coefficient determines the effect of fusion. It was proved that the predictions are more accuracy when the 3D convolution model obtains a higher weight through a series of experiments. But the results of SVM-based fusion performs better than fusion by averaging. Therefore, a SVM classifier is trained to fuse the video-level softmax scores from multiple model.

\subsection{Training}

During training, the three models mentioned above training parameters separately. It has a softmax classification layers on top of the last fc layer ( fully connected layer ):

$$
y_{i}=\frac{e^{X_{i}}}{\sum_{i=1}^{n} e^{X_{i}}}, i \epsilon[1, N]
$$

where $X_{i}$ is the output of the ith neuron in the last fc layer of the network. $\mathrm{N}$ is the total class number of action recognition classification.
In the training stage, cross entropy loss is calculated according to the output of the last fc layer with the true value. the final loss function is:

$$
L=-\sum z_{i} \ln y
$$

Where $Z_{i}$ is the real classification results. A gradient-based optimization method Stochastic gradient descent (SGD) is used to learn the model parameters.

Data augmentation can generate diverse training samples and prevent severe over-fitting. Some traditional methods are used for data augmentation: random cropping and horizontal flipping corner cropping and scale jittering. Polynomial kernel function is used as a kernel function in the SVM. The model based on 3D convolutional was pre-trained on the sports1M dataset. And the model based on two-stream was pre-trained on ImageNet.

\section{EXPERIMENTAL RESULTS}

In this section, the evaluation datasets and evaluation protocol are firstly introduced. Then, a series of experiments are conducted to prove the superiority of the proposed method. Finally, the proposed method is compared with other recognition models on UCF-101[28].

\subsection{Datasets and evaluation protocol}

Experiments are conducted on UCF-101[28]. UCF-101 is a trimmed video dataset, consisting of realistic web videos with diverse forms of camera motion and illumination. It contains 13,320 videos with 101 action classes. Three training/test splits are provided. And each split in UCF-101 includes about $9.5 \mathrm{~K}$ training and $3.7 \mathrm{~K}$ test videos. The mean average accuracy over three testing splits is finally reported for the comparison with other action recognition approaches. And the $\mathrm{K}$ is set to 3 and 16 frames are chosen to be the input of proposed method to achieve a balance in the calculated amount and accuracy during test.

\subsection{Video-level recognition result}

Table. 1 shows the performance compared with 3D convolutional action recognition methods. Among these method, only RGB frames are used as inputs for a fair comparison. Note that, P3D ResNet[24], I3D(only RGB)[5], ResNeXt101(16f)[14], MiCT-Net(Mixed Convolutional Tube Net)[38] and original C3D[7], these are considered segment-level action recognition methods because these methods are only relevant to partial time snippets on the video. All of these methods mentioned above take 16 frames as input for training except MiCT-Net(take 75 frames as input). The results of proposed method(Video-level 3D convolution) achieve the best performance among all 3D CNNs based methods, and verify that modeling video-level model is crucial for better understanding of action in videos.

Long-term video-level 3D convolutional action recognition methods with only RGB as inputs, e.g. LTC(Long-term Temporal Convolutions)[33], is alse compared. The proposed method still outperforms LTC with a $9.3 \%$ gain in accuracy. 
Table 1: Comparation with the 3D convolutional based method. "*" stands for the video-level action recognition method.

\begin{tabular}{ccc}
\hline Command & A Number & Comments \\
\hline I3D(only RGB) [5] & 16 & $84.5 \%$ \\
P3D ResNet [24] & 16 & $88.6 \%$ \\
MiCT-Net [38] & 75 & $88.9 \%$ \\
ResNeXt-101(16f) [14] & 16 & $90.7 \%$ \\
\hline LTC(only RGB) (100f) [33]* & 100 & $82.4 \%$ \\
\hline C3D [7] & 16 & $87.8 \%$ \\
Our Method(Video-level 3D convolution model)* & $\mathbf{1 6}$ & $\mathbf{9 1 . 7 \%}$ \\
\hline
\end{tabular}

Table 2: Exploration of multi-model fusion on the UCF-101 dataset.

\begin{tabular}{ccccc}
\hline Modality & split1 & split2 & split3 & Ave \\
\hline S-TSN(spacial stream from TSN[35]) & $86.0 \%$ & $85.0 \%$ & $84.6 \%$ & $85.2 \%$ \\
T-TEN(temporal stream from TSN[35]) & $87.6 \%$ & $90.2 \%$ & $91.3 \%$ & $89.7 \%$ \\
V-3D & $92.6 \%$ & $90.8 \%$ & $91.7 \%$ & $91.7 \%$ \\
TSN[35] & $93.5 \%$ & $94.2 \%$ & $94.5 \%$ & $94.1 \%$ \\
\hline VMF(video-level multi-model fusion for action recognition) & $\mathbf{9 7 . 3 \%}$ & $\mathbf{9 8 . 0 \%}$ & $\mathbf{9 7 . 6 \%}$ & $\mathbf{9 7 . 6 \%}$ \\
\hline
\end{tabular}

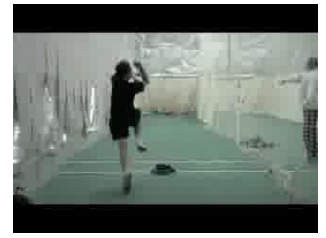

CricketBowling

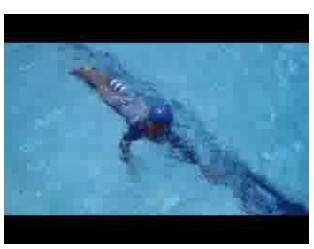

FrontCraw1

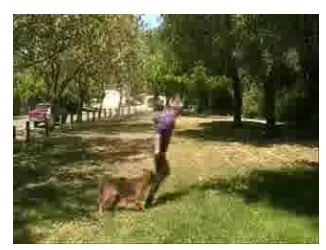

WalkingWithDog
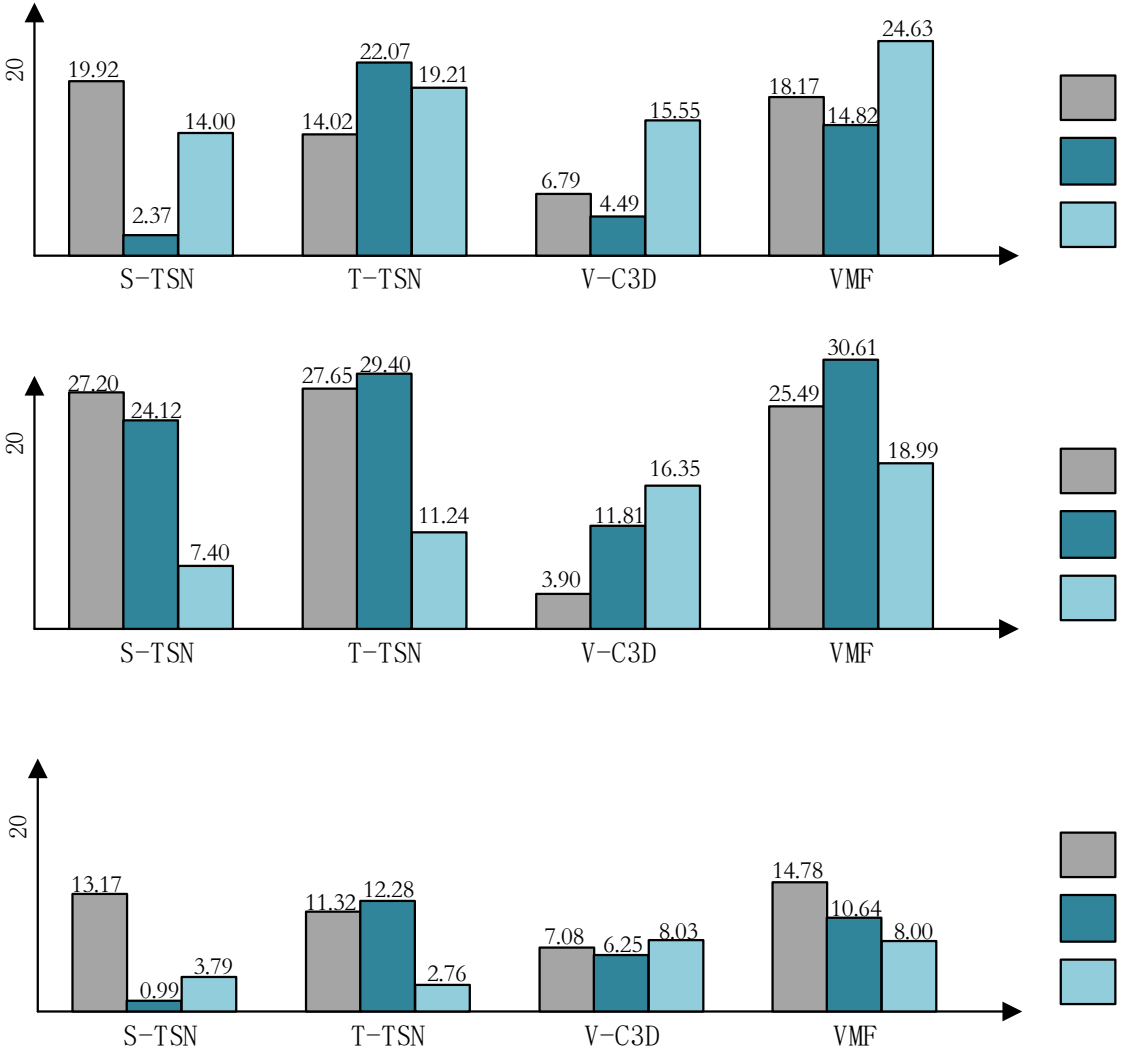

WalkingWithDog

TaiChi

Kayaking

Figure 3: The input video with the correct label(left), and the results of the spatial stream, temporal stream, video-level 3D convolution model, and multi-model(right). The scores of predicted action which are come from three separate models are displayed. 
At the same time, the proposed method has the same number of training parameters compared to the original C3D [7]. But the proposed video-level action recognition accuracy is $4 \%$ higher than original C3D model(segment-level). Therefore, it is a lightweight and efficient action recognition method to overcome the confusion caused by partial observation.

\subsection{Multi-model fusion recognition result}

In this subsection, five set of parallel experiments were conducted to measure the performance of multi-model fusion method.

- S-TSN(spacial stream from TSN[35]): a 2D convolutional network which take only single RGB as input.

- T-TSN ( Temporal stream from TSN[35]): a 2D convolutional network which takes only optical flow stacks as input.

- V-3D (Video-level C3D): a 3D convolutional network which takes continuous frames as input.

- TSN[35]: a 2D convolutional network which takes single RGB and stated optical flow as input.

- VMF (video-level multi-model fusion for action recognition): a network combined $2 \mathrm{D}$ convolutional and $3 \mathrm{D}$ convolutional which takes single RGB, stated optical flow and continuous frames as input.

The experimental data from TSN[35] which fuses S-TSN( spacial stream) and T-TSN( temporal stream) represent the direct complementariness between temporal information and spacial information. And the experimental data from VMF(video-level multi-model fusion for action recognition) represent the complementariness between temporal information, spatial information and spatio-temporal information.

Figure. 3 provides case studies that illustrate the complementariness among the temporal, spatial and spatio-temporal information. Each set of videos are followed by four sets of experiment data: S-TSN, T-TSN, V-3D, VMF(from left to right). The black label represent the ground truth, which is the correct classification of the video.

As shown in Figure. 3, the real label of the first video is "Cricket bowling", and the predictions of S-TSN and T-TSN are wrong. Spatio-temporal information provides important clues for action recognition. So the correct prediction results are obtained by the method of V-3D which takes full advantages of spatio-temporal information. In this case, the VMF method corrects the result by fully applying multiple information include the spatio-temporal information. For the second video, the prediction of T-TSN is true, because the temporal information acquired by T-TSN is especially important. Due to the lack of guidance for motion information, only general action types "swimming" can be identified. However it is difficult to distinguish between breast stroke and front crawl for S-TSN. And the result of V-3D is more deviated in the case that spatio-temporal information it acquired is not specific. But VMF gets the right answer because it acquire more comprehensive information. For the last video, the result of S-TSN is correct. One possible reason is the
RGB image contains clues such as dog which is a key point in space dimension. Some details of the space dimension are often ignored in the temporal stream and 3D convolutional networks, which plays an important role in the recognition of some actions. The proposed method which combine various information still performs well.

The results of the experiments on the UCF-101 dataset are shown in Table. 2. It is obvious that VMF is much better than the single model and the two stream model. Besides, the results here are all video-level recognition results, and the number of segment is set to 3 for all methods. Weighted summation method is used to fuse the scores of spacial stream and temporal stream in TSN here(spacial stream:temporal stream $=1: 1.5)$. The experimental results show that VMF is very effective. VMF improves the accuracy of V-3D by $5.9 \%$, and improves the accuracy of TSN by $3.5 \%$.

Therefore, it can be concluded that the methods that obtains the information of only one dimension( spacial, temporal or spatio-temporal ) such like S-TSN, T-TSN and V-3D have their limitations in the acquisition of information. The proposed video-level multi-model fusion method performs well which validate the complementariness among spatial, temporal and spatio-temporal information.

In addition, different fusion methods have been tried. The softmax scores of the three methods(S-TSN, T-TSN and $\mathrm{V}-3 \mathrm{D})$ are combined using weighted summation method or SVM. In the experiments, the best effect is achieved when the ratio is $1: 1.2: 1.5(\mathrm{~S}-\mathrm{TSN}: \mathrm{T}-\mathrm{TSN}: \mathrm{V}-3 \mathrm{D})$, which is better than the average fusion (1:1:1). Table. 3 shows SVM-based fusion of softmax scores performs better than weighted summation method.

\subsection{Comparison with the State of the Art}

In the end, a comparison of the performance of VMF and previous state-of-the-art methods is shown in Table. 4. The mean average accuracy on the three testing splits on UCF-101 of the proposed method is reported.

Spacial and temporal information has been proven helpful for action recognition in many previous works. Several two-stream based methods are presented to fuse the two networks over the appearance and motion, e.g. Two stream[34], TSN[35], Four-Stream[1], DTPP[41]. Regarding two stream based methods with the best accuracy up to $96.2 \%$ on UCF-101. The proposed method outperforms previous two stream based best method by $1.4 \%$. Thereinto, TSN, Four-Stream and DTPP are also video-level representation learning method.

Compared to LSTM based method, e.g. LSTM with 30 Frame Unroll(Flow+RGB)[22] and Multimodal Keyless Attention Fusion for Video Classification(Flow+RGB)[37]. The proposed method is far superior to LSTM with 30 Frame Unroll(Flow+RGB). And is $2.8 \%$ higher than Multimodal Keyless Attention Fusion for Video Classification.

Similarly, compared to 3D CNN based method, e.g. C3D[7], LTC[33], P3D ResNet[24], ResNeXt[14], R(2+1)D[8], the proposed method outperforms the best method by $2.6 \%$. And 
Table 3: The results of different fusion methods.

\begin{tabular}{cc}
\hline Fusion method & Performance \\
\hline Weighted fusion(1:1.2:1.5) & $97.4 \%$ \\
SVM & $\mathbf{9 7 . 6 \%}$ \\
\hline
\end{tabular}

Table 4: Comparison with the state-of-the-art on UCF101 (mean accuracy across 3 splits).

\begin{tabular}{ccc}
\hline Modality & dim & Performance \\
\hline Two stream[34] & 2 & $88.0 \%$ \\
TSN[35] & 2 & $94.2 \%$ \\
Four-Stream+IDT[1] & 2 & $96.0 \%$ \\
DTPP+iDT[41] & 2 & $96.2 \%$ \\
\hline LSTM with 30 Frame Unroll(Flow+RGB) [22] & 2 & $88.6 \%$ \\
Multimodal Keyless Attention Fusion for Video Classification(Flow+RGB) [37] & 2 & $94.8 \%$ \\
\hline C3D+iDT[7] & 3 & $90.1 \%$ \\
LTC(Flow+RGB+IDT)[33] & 3 & $92.7 \%$ \\
P3D ResNet + iDT[24] & 3 & $93.7 \%$ \\
ResNeXt-101 (64f)[14] & 3 & $94.5 \%$ \\
R(2+1)D[8] & 3 & $95.0 \%$ \\
\hline Two-stream MiCT-Net [38] & 3 & $95.6 \%$ \\
VMF & 3 & $\mathbf{9 7 . 6 \%}$ \\
\hline
\end{tabular}

the accuracy of proposed method is $2.6 \%$ higher than MiCTNet[38] which integrates 2D CNNs with the 3D convolution module too.

The proposed method significantly enhances performance which indicates the complementariness among spatial, temporal and spatio-temporal information plays an important part in the action recognition. Due to the fusion of multiple models, the proposed method has no advantage in computing speed.

Moreover, we did not undergo pre-training on big dataset Kinetics due to time and experimental machines. Compared to other action recognition method, our results achieves stateof-the-art results of $97.6 \%$ on UCF101 under the conditions without pre-training on Kinetics.

One reason is that the Kinetics dataset was not successfully downloaded because of the limitations of the Internet. Another is that due to the limit of the experimental environment, it is time consuming to train the model on Kinetics using 1 GPU.

\section{CONCLUSION}

In this paper, video-level multi-model fusion for action recognition is proposed which combines $2 \mathrm{D}$ convolution with $3 \mathrm{D}$ convolution to make full use of temporal, spatial and spatiotemporal information. It is confirmed that the video-level recognition methods preform better than segment-level recognition methods. And the complementariness among spatial, temporal and spatio-temporal information indicates that it is worthwhile to take Multi-model fusion. At the same time, the proposed video-level Multi-model Fusion method achieves good performance on UCF-101 without pre-trained on Kinetics. Kinetics pre-training helps significantly more than ImageNet and some other datasets. Because of the limitation of conditions, the Kinetics is not used to pre-train in the proposed method.

The optical flow provides more detailed motion information which is very beneficial for action recognition. Because the calculation of optical flow is time consuming the flow-based descriptors is proposed for action recognition in recent years. But the flow-based descriptors are not perform as well as the real optical flow in action recognition. Therefore, a better way remains to be proposed.

\section{ACKNOWLEDGEMENTS}

This work is supported in part by the China Scholarship Council(201806455020), Open Research Fund of Beijing Key Laboratory of Big Data Technology for Food Safety, Beijing Technology and Business University, the Natural Science Foundation of Hebei Province(F2018511002), the National Natural Science Foundation of China (Grant No. 61877002), NSF under grants III-1526499, III-1763325, III-1909323, SaTC1930941, and CNS-1626432

\section{REFERENCES}

[1] Hakan Bilen, Basura Fernando, Efstratios Gavves, and Andrea Vedaldi. 2016. Action Recognition with Dynamic Image Networks. IEEE Transactions on Pattern Analysis and Machine Intelligence PP, 99 (2016), 1-1.

[2] Zhe Wang Yu Qiao Bowen Zhang, Limin Wang and Hanli Wang. 2018. Real-time action recognition with deeply transferred motion vector cnns. IEEE Transactions on Image Processing (2018), 1, $3,4,8$. 
[3] Andrés Bruhn, Joachim Weickert, and Christoph Schnörr. 2005. Lucas/Kanade Meets Horn/Schunck: Combining Local and Global Optic Flow Methods. International Journal of Computer Vision 61, 3 (01 Feb 2005), 211-231. https://doi.org/10.1023/B:VISI 0000045324.43199 .43

[4] Daniel J. Butler, Jonas Wulff, Garrett B. Stanley, and Michael J. Black. 2012. A Naturalistic Open Source Movie for Optical Flow Evaluation. In Computer Vision - ECCV 2012, Andrew Fitzgibbon, Svetlana Lazebnik, Pietro Perona, Yoichi Sato, and Cordelia Schmid (Eds.). Springer Berlin Heidelberg, Berlin, Heidelberg, $611-625$.

[5] Joao Carreira and Andrew Zisserman. 2017. Quo Vadis, Action Recognition? A New Model and the Kinetics Dataset. (2017) 4724-4733.

[6] Alexey Dosovitskiy, Philipp Fischer, Eddy Ilg, Philip Hausser Caner Hazirbas, Vladimir Golkov, Patrick van der Smagt, Daniel Cremers, and Thomas Brox. 2015. FlowNet: Learning Optical Flow With Convolutional Networks. In The IEEE International Conference on Computer Vision (ICCV).

[7] Tran Du, Lubomir Bourdev, Rob Fergus, Lorenzo Torresani, and Manohar Paluri. 2015. Learning Spatiotemporal Features with 3D Convolutional Networks. IEEE International Conference on Computer Vision (2015), 4489-4497.

[8] Tran Du, Heng Wang, Lorenzo Torresani, Jamie Ray, Yann Lecun, and Manohar Paluri. 2017. A Closer Look at Spatiotemporal Convolutions for Action Recognition. (2017).

[9] Lijie Fan, Wenbing Huang, Chuang Gan, Stefano Ermon, Boqing Gong, and Junzhou Huang. 2018. End-to-End Learning of Motion Representation for Video Understanding. In The IEEE Conference on Computer Vision and Pattern Recognition (CVPR).

[10] Christoph Feichtenhofer, Axel Pinz, and Andrew Zisserman. 2016 Convolutional Two-Stream Network Fusion for Video Action Recognition. (2016), 1933-1941.

[11] P Felzenszwalb, D McAllester, and D Ramanan. 2008. A discriminatively trained, multiscale, deformable part model. IEEE Conference on Computer Vision and Pattern Recognition (2008), $1 \mathrm{C} 8$.

[12] Ruohan Gao, Bo Xiong, and Kristen Grauman. 2018. Im2Flow: Motion Hallucination From Static Images for Action Recognition In The IEEE Conference on Computer Vision and Pattern Recognition (CVPR).

[13] Alex Graves. 1997. Long Short-Term Memory. Neural Computation 9, 8 (1997), 1735-1780.

[14] Kensho Hara, Hirokatsu Kataoka, and Yutaka Satoh. 2017. Can Spatiotemporal 3D CNNs Retrace the History of 2D CNNs and ImageNet? (2017).

[15] Berthold K.P. Horn and Brian G. Schunck. 1981. Determining optical flow. Artificial Intelligence 17, 1 (1981), 185 - 203.

[16] Eddy Ilg, Nikolaus Mayer, Tonmoy Saikia, Margret Keuper, Alexey Dosovitskiy, and Thomas Brox. 2017. FlowNet 2.0: Evolution of Optical Flow Estimation With Deep Networks. In The IEEE Conference on Computer Vision and Pattern Recognition (CVPR).

[17] Sergey Ioffe and Christian Szegedy. 2015. Batch Normalization Accelerating Deep Network Training by Reducing Internal Covariate Shift. International Conference on International Conference on Machine Learning. (2015), 448-456.

[18] Yang M et al. Ji S, Xu W. 2013. 3D Convolutional Neural Networks for Human Action Recognition[J]. IEEE Transactions on Pattern Analysis and Machine Intelligence (2013), 35(1):221-231.

[19] H Kaiming, Zhang Xiangyu, and Ren Shaoqing. 2016. Deep residual learning for image recognition. IEEE Conference on Computer Vision and Pattern Recognition (2016).

[20] Andrej Karpathy, George Toderici, Sanketh Shetty, Thomas Leung, Rahul Sukthankar, and Fei Fei Li. 2014. Large-Scale Video Classification with Convolutional Neural Networks. (2014), 17251732 .

[21] Christian Heipke Moritz Menze and Andreas Geiger. Joint. 2015. $3 \mathrm{~d}$ estimation of vehicles and scene flow. In ISPRS Workshop. In Image Sequence Analysis (ISA).

[22] Yue Hei Ng, Matthew Hausknecht, Sudheendra Vijayanarasimhan, Oriol Vinyals, Rajat Monga, and George Toderici. 2015. Beyond Short Snippets: Deep Networks for Video Classification. Computer Vision and Pattern Recognition (2015), 4694-4702.

[23] Yue Hei Ng, Matthew Hausknecht, Sudheendra Vijayanarasimhan, Oriol Vinyals, Rajat Monga, and George Toderici. 2015. Beyond Short Snippets: Deep Networks for Video Classification. Computer Vision and Pattern Recognition (2015), 4694-4702.
[24] Zhaofan Qiu, Ting Yao, and Tao Mei. 2017. Learning SpatioTemporal Representation with Pseudo-3D Residual Networks. IEEE International Conference on Computer Vision (ICCV). (2017), 5534-5542.

[25] Hamidreza Rabiee, Hossein Mousavi, Moin Nabi, and Mahdyar Ravanbakhsh. 2017. Detection and localization of crowd behavior using a novel tracklet-based model. International Journal of Machine Learning and Cybernetics (2017), 1-12.

[26] Anurag Ranjan and Michael J. Black. 2017. Optical Flow Estimation Using a Spatial Pyramid Network. In The IEEE Conference on Computer Vision and Pattern Recognition (CVPR).

[27] Laura Sevilla-Lara, Yiyi Liao, Fatma Guney, Varun Jampani, Andreas Geiger, and Michael J. Black. 2019. On the Integration of Optical Flow and Action Recognition. In Pattern Recognition, Thomas Brox, Andres Bruhn, and Mario Fritz (Eds.). Springer International Publishing, 281-297.

[28] Khurram Soomro, Amir Roshan Zamir, and Mubarak Shah. 2012. UCF101: A Dataset of 101 Human Actions Classes From Videos in The Wild. Computer Science (2012).

[29] Deqing Sun, Stefan Roth, J. P. Lewis, and Michael J. Black. 2008. Learning Optical Flow. In Computer Vision - ECCV 2008, David Forsyth, Philip Torr, and Andrew Zisserman (Eds.). Springer Berlin Heidelberg, Berlin, Heidelberg, 83-97.

[30] Deqing Sun, Xiaodong Yang, Ming-Yu Liu, and Jan Kautz. 2018. PWC-Net: CNNs for Optical Flow Using Pyramid, Warping, and Cost Volume. In The IEEE Conference on Computer Vision and Pattern Recognition (CVPR).

[31] Christian Szegedy, Wei Liu, Yangqing Jia, Pierre Sermanet, Scott Reed, Dragomir Anguelov, Dumitru Erhan, Vincent Vanhoucke, and Andrew Rabinovich. 2014. Going Deeper with Convolutions. (2014), 1-9.

[32] Yicong Tian, Rahul Sukthankar, and Mubarak Shah. 2013. Spatiotemporal Deformable Part Models for Action Detection. Computer Vision and Pattern Recognition (2013), 2642-2649.

[33] G Varol, I Laptev, and C Schmid. 2018. Long-term Temporal Convolutions for Action Recognition. IEEE Transactions on Pattern Analysis and Machine Intelligence PP, 99 (2018), 1-1.

[34] Limin Wang, Yu Qiao, and Xiaoou Tang. 2015. Action Recognition with Trajectory-Pooled Deep-Convolutional Descriptors. (2015), 4305-4314.

[35] Limin Wang, Yuanjun Xiong, Zhe Wang, Yu Qiao, Dahua Lin, Xiaoou Tang, and Luc Van Gool. 2016. Temporal Segment Networks: Towards Good Practices for Deep Action Recognition. Acm Transactions on Information Systems 22, 1 (2016), 20-36.

[36] Chao-Yuan Wu, Manzil Zaheer, Hexiang Hu, R. Manmatha, Alexander J. Smola, and Philipp Kr?henbhl. 2018. Compressed Video Action Recognition. In The IEEE Conference on Computer Vision and Pattern Recognition (CVPR).

[37] Long X, Gan C, de Melo G, Liu X, Li Y, Li F, and Wen S. 2018. Multimodal keyless attention fusion for video classification. AAAI (2018).

[38] Zhou Y, Sun X, and Zha Z J. 2018. MiCT: Mixed 3D/2D Convolutional Tube for Human Action Recognition. IEEE Conference on Computer Vision and Pattern Recognition (2018), 449-458.

[39] C. Zach, T. Pock, and H. Bischof. 2007. A Duality Based Approach for Realtime TV-L1 Optical Flow. In Pattern Recognition, Fred A. Hamprecht, Christoph Schnörr, and Bernd Jähne (Eds.). Springer Berlin Heidelberg, Berlin, Heidelberg, 214-223.

[40] Bolei Zhou, Aditya Khosla, Agata Lapedriza, Aude Oliva, and Antonio Torralba. 2016. Learning Deep Features for Discriminative Localization. (June 2016).

[41] Jiagang Zhu, Wei Zou, and Zheng Zhu, 2017. End-to-end Videolevel Representation Learning for Action Recognition. arXiv (2017). 